\section{P24 ASSOCIATION BETWEEN ANTIBIOTIC PRESCRIBING AND DEPRIVATION IN WALES: A MULTILEVEL ANALYSIS}

${ }^{1} \mathrm{~V}$ Adekanmbi*, ${ }^{1} \mathrm{~A}$ Smith, ${ }^{1} \mathrm{D}$ Farewll, ${ }^{1} \mathrm{H}$ Jones, ${ }^{1} \mathrm{~S}$ Paranjothy, ${ }^{2} \mathrm{P}$ Routledge, ${ }^{3} \mathrm{~N}$ Francis. ${ }^{1}$ Division of Population Medicine, Cardiff University, Cardiff, UK; ${ }^{2}$ All Wales Therapeutics and Toxicology Centre, Academic Centre, Cardiff University, Cardiff, UK; ${ }^{3}$ Centre for Trials Research, School of Medicine, Cardiff University, Cardiff, UK

\subsection{6/jech-2018-SSMabstracts.150}

Background Antibiotic prescription rates have decreased steadily since 2011 in Wales. The most recent Welsh Antimicrobial Resistance Programme (WARP) report on antibiotic use in primary care found significant variations between Health Boards in gross antibiotic use in 2014. It is however not clear whether there is widening gap in prescribing volumes between the most and least deprived neighbourhood as well as hospitals across the country. The aim of this study was to evaluate the association between socioeconomic deprivation and antibiotic prescribing volumes in Wales.

Methods Welsh General Practitioner (GP) antibiotic prescribing data for years 2013 to 2016 for patients' resident in Wales were extracted from the Secure Anonymised Information Linkage GP tables. Deprivation was assessed by linking prescribing events to the Welsh Index of Multiple Deprivation score for the patient's neighbourhood area. The association between deprivation area and antibiotic prescribed (items per 1000 persons per day) was stratified according to the patient's age, sex, prescription year and antibiotic class. A three-level multilevel Poisson regression model of 1.58 million patients nested within 349 GP practices, nested with 67 GP clusters, was specified to assess the associations.

Results Just over 7.97 million antibiotic items were prescribed between 2013 and 2016. Patients in the most deprived WIMD quintile had an overall prescription rate that was 25.2\% higher than those in the least deprived WIMD quintile. The final model revealed that residing in the most deprived WIMD quintile (incidence rate ratio $[\mathrm{IRR}]=1.1769$, 95\% confidence interval [CI] 1.1768 to 1.1770 , being female $(\mathrm{IRR}=1,2699$, 95\% CI 1.2698 to 1.2700$)$, being aged $\geq 90$ (IRR $=2.0687$, 95\% CI 2.0683 to 2.0690 ), and prescription year being 2013 were associated with significantly higher rate of antibiotics prescription. There were significant primary cares clustering of antibiotics prescription in Wales.

Conclusion This study provides evidence that patients in areas of higher socioeconomic deprivation are more likely to be prescribed antibiotics in primary care in Wales. Population health prevention strategies aimed at reducing high antibiotic prescription rates should consider targeting areas of high deprivation.

\section{P25 SUPPORTIVE CARE NEEDS OF YOUNG ADULTS WITH A STOMA: A QUALITATIVE STUDY}

${ }^{1} \mathrm{KP}$ Polidano ${ }^{*},{ }^{2,3} \mathrm{ADF}$ Farmer, ${ }^{1} \mathrm{BB}$ Bartlam, ${ }^{1} \mathrm{BS}$ Saunders. ${ }^{1}$ Research Institute for Primary Care and Health Sciences, Keele University, Keele, UK; ${ }^{2}$ Institute of Applied Clinical Sciences, Keele University, Keele, UK; ${ }^{3}$ Department of Gastroenterology, University Hospitals of North Midlands, Stoke-on-Trent, UK

\subsection{6/jech-2018-SSMabstracts.151}

Background Living with a stoma can have a significant psychological impact, especially for individuals with inflammatory bowel disease (IBD) who may need ostomy surgery at a relatively young age. Previous research indicates that body image, sexual relationships, lifestyle and self-identity may all be affected as a result of stoma formation. While extensive research has investigated these psychological challenges, relatively little is known about the supportive care needs of younger stoma patients and how these are to be met. This presentation will outline the preliminary findings of a qualitative study that addresses this knowledge gap.

Methods In-depth interviews are being conducted with young adults (18-29 years) who have a stoma as a result of IBD, and a range of healthcare professionals involved in their care pathway (including surgeons, gastroenterologists, IBD nurses, stoma care nurses, and primary care physicians). A combination of constructivist grounded theory and narrative inquiry are informing data-collection and analysis.

Results Emerging findings suggest that young adults perceive a general lack of psychological support throughout their illness trajectory. The need for more holistic stoma care services, better accessibility to psychosocial interventions, increased signposting to other support avenues, as well as better coordination and consistency of care are highlighted by patients and healthcare professionals alike. Young adults also emphasise the importance of age-appropriate care, expressing a preference for peer support, particularly online, as a support tool.

Conclusion This ongoing study confirms the importance of refocusing research attention towards the supportive care needs of this patient-group. Findings will have implications for improving access to care and support for young adults with a stoma, with the ultimate goal of effectively identifying and managing psychological problems among this population.

\section{P26 EARLY MOTHERHOOD: A QUALITATIVE STUDY EXPLORING EXPERIENCES OF ADOLESCENT MOTHERS IN THE HOHOE MUNICIPALITY OF GHANA}

${ }^{1,2} \mathrm{~S}$ Gbogbo*, ${ }^{1} \mathrm{MA}$ Ayanore, ${ }^{3} \mathrm{Y}$ Enuameh, ${ }^{2} \mathrm{C}$ Schweppe. ${ }^{1}$ School of Public Health, University of Health and Allied Sciences, Ho, Ghana; ${ }^{2}$ Institute of Education, Johannes Gutenberg University Mainz, Mainz, Germany; ${ }^{3}$ School of Public Health, Kwame Nkrumah University of Science and Technology, Kumasi, Ghana

\subsection{6/jech-2018-SSMabstracts. 152}

Background Each year between 14 and 15 million adolescent girls give birth, accounting for more than $10 \%$ of births globally. Motherhood is an exciting time for every woman and the society at large and also seen as a significant part of any woman's identity but that is often not the case for the adolescent mother. Adolescent motherhood can be a time of mixed feelings as it occurs at a critical time of their lives. This study examined challenges adolescent mothers face during early motherhood and strategies to improve early motherhood for better social and health outcomes.

Methods Based on a phenomenological perspective, this qualitative study was conducted in the Hohoe Municipality where purposive sampling was used to recruit study participants. The process of data gathering included, 6 focus group discussions held with adolescent mothers, 20 in-depth interviews with pregnant adolescents, 6 in-depth interviews conducted with midwives and traditional birth attendants (TBAs). Using thematic analysis, recorded data were transcribed and manually coded inductively and deductively for themes.

Results A total participants of 60 teenage Junior High School dropouts were interviewed, out of which 10 mothers were in school after initial dropout. The average age of participants was 15 years. Other participants included 3 midwives and 3 